\title{
Using Negative Pressure Wound Therapy in the Treatment of Wounds After Fasciotomy in Acute Compartment Syndrome - Retrospective Case Series Study
}

\author{
Jarosław P. Miszczuk ${ }^{1,2 *}$, Maria Błońska-Staniec ${ }^{2}$, Anna Michalska ${ }^{1}$, Bartosz Stemplewski ${ }^{2}$ \\ 1 Collegium Medicum, The Jan Kochanowski University, Kielce, Poland \\ 2 Department of Vascular Surgery, Provincial Hospital, Kielce, Poland \\ * Correspondence: Department of Vascular Surgery, Provincial Hospital, Grunwaldzka 45, 25-736 Kielce; \\ Tel.: 4136714 02, 500049861
}

\begin{abstract}
:
Background and objectives: Acute compartment syndrome (ACS) is an emergency condition of the lower limb in which prophylactic fasciotomy is required to prevent complications. A negative pressure wound therapy (NPWT) used to the treatment of fasciotomy wounds provide beneficial clinical results. This study aimed to exchange the authors' experience of using the NPWT installation system on the lower limb wounds after fasciotomy in ACS. Materials and Methods: This is a retrospective study enrolled consecutive patients with ACS, who underwent fasciotomy and was treated with the NPWT installation system at Department of Vascular Surgery, Provincial Hospital in Kielce from April 2016 to July 2017. Results: The study enrolled 15 patients with a diagnosis of ACS (87\% men, mean age 65 years old). An open four-compartment fasciotomy $(87 \%)$ or two-compartment fasciotomy (13\%) was performed. The NPWT was applied on the first day after fasciotomy in $87 \%$ of patients. Therapy was initiated by the negative pressure of $125 \mathrm{~mm} \mathrm{Hg}$, which maintained at this level until the therapy was finalized. In $80 \%$ of patients, the vacuum-assisted wound closure (VAC) dressing changes were performed every 3 days. The first approximation of fasciotomy wounds margins occurred on the 4 th day after surgery among $67 \%$ of individuals. The average time of using VAC on fasciotomy wounds was 9 days. The average time to definitive closure edges of fasciotomy wounds was 12 days. The average time of hospital stay was 17 days. Conclusions: Our experience indicates the legitimacy of using NPWT in wound treatment after fasciotomy in ACS. The NPWT enables faster primary closure of wounds, reduces edema, as well as decreases hospitalization time.
\end{abstract}

Keywords: acute compartment syndrome; negative pressure wound therapy; vacuum-assisted wound closure; fasciotomy

\section{Introduction}

Acute compartment syndrome (ACS) is an emergency post-traumatic or post-operative condition of the lower limb due to post-ischemic reperfusion. The most common types of trauma related to the development of ACS are tibial shaft fracture, crush trauma and soft-tissue injury. Other causes leading to the development of ACS are reperfusion injury after acute limb ischemia, injuries of the arterial and venous system, and bleeding into fascial compartments.

In this condition, the local reduction of blood flow, tissue hypoxia, nerve ischemia, muscle damage, necrosis of the soft tissues in consequence of increased intra-compartmental pressure may occur [1-3]. To prevent complications leading to permanent disabilities, a prophylactic fasciotomy is 
applied. This method is associated with the risk of surgical site infection (SSI), injuries to the peroneal nerve and the necessity of prolonged patient hospitalization [4]. In order to avoid complications, the wound should be closed as soon as possible. An extensive wound has posed a significant nursing problem, associated with an increased risk of SSI and worsened the healing process. Moreover, the use of skin grafting to the closure of the wound may lead to the formation of hypertrophic scars, both in the site of removing and transplanting skin area [5-6].

In turn, using negative pressure wound therapy (NPWT), also known as vacuum-assisted closure (VAC) dressing, to the treatment of surgical wounds results in reduction of local edema, improves blood circulation in the wound, leads to the early development of granulation tissue and decreases the incidence of SSI. Additionally, NPWT reduces secondary amputations, provides a better cosmetic effect and contributes shorter hospitalization periods. A systematic review of the literature has provided that NPWT therapy enables faster primary closure of wounds [7-9].

This study aimed to exchange the authors' experience of using the NPWT installation system on the lower limb wounds after fasciotomy in ACS.

\section{Materials and Methods}

The retrospective case series study was conducted on consecutive patients aged $\geq 45$ years with ACS, who underwent fasciotomy and was treated with the NPWT installation system at Department of Vascular Surgery, Provincial Hospital in Kielce from April 2016 to July 2017. The VAC therapy with using a special dressing sponge dedicated to the RENASYS EZ Plus (Smith \& Nephew) or Comfort device were applied on fasciotomy wounds. Patients' characteristics were obtained retrospectively from in-hospital records.

Among analyzed patients, wounds occurred on the anterior surface of lower limbs on the lateral or/and medial side. The NPWT was applied after therapeutic fasciotomy to reduce edema, decrease infection risk and evacuate excess of fluids. During the dressing application, a smaller size of sponge in relation to the wound was chosen. This technique does not stretch the wound, which provides a beneficial effect on the primary closure process in any subsequent dressing replacement. In the time of consecutive hospital visits, the progress of the fasciotomy wound healing was monitored by follow-up examinations. At follow up examination physicians collected patient interviews, checked the pulse on lower limbs, made USG doppler and assessed the ankle-brachial index (ABI).

Written informed consent for vascular surgery and examination was obtained from each engaged individual.

\section{Results}

The study included 15 patients with a diagnosis of ACS (87\% men, mean age 65 years old) admitted to the Department of Vascular Surgery, Provincial Hospital. The majority of patients with ischemia-reperfusion edema of the lower limb after the restoration of blood supply were enrolled in this study. In $87 \%$ of patients, an open four-compartment fasciotomy was performed. Two patients (13\%) had an open fasciotomy with two-compartment [Fig. 1,2]. 


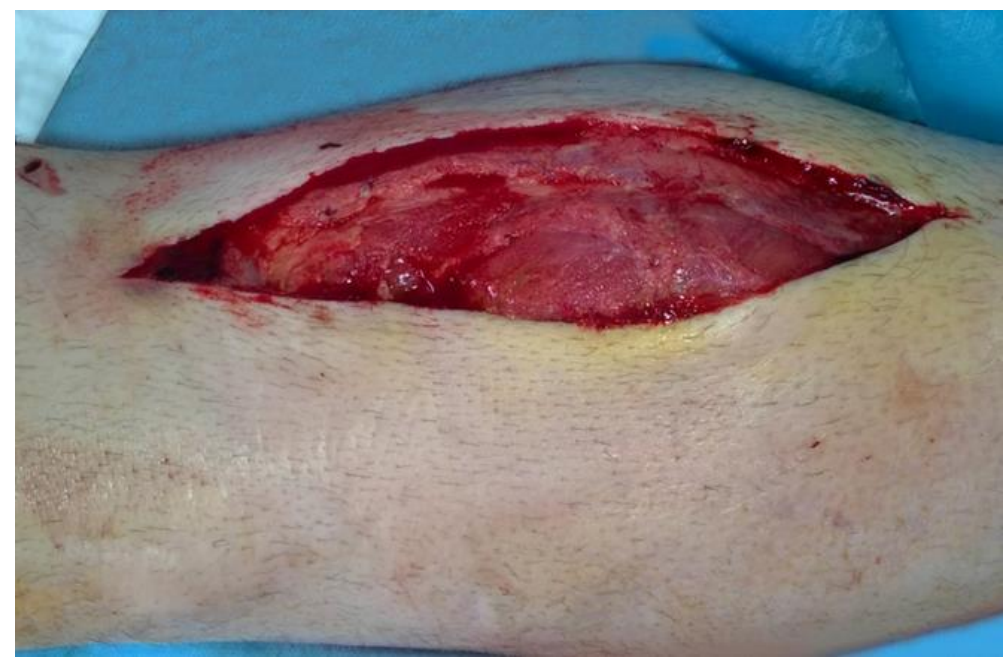

Figure 1. Open fasciotomy of the lower limb - medial cut.

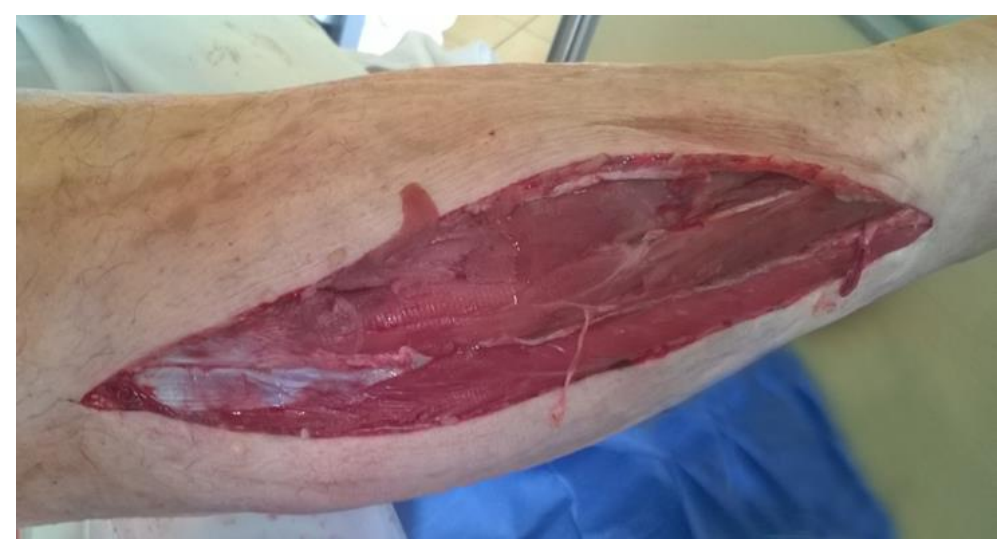

Figure 2. Open fasciotomy of the lower limb - lateral cut.

The NPWT was applied on the first day after fasciotomy in $87 \%$ of patients [Fig. 3]. Therapy was initiated by the negative pressure of $125 \mathrm{~mm} \mathrm{Hg}$, which maintained at this level until the therapy was finalized. In $80 \%$ of patients, the VAC dressing changes were performed every 3 days.

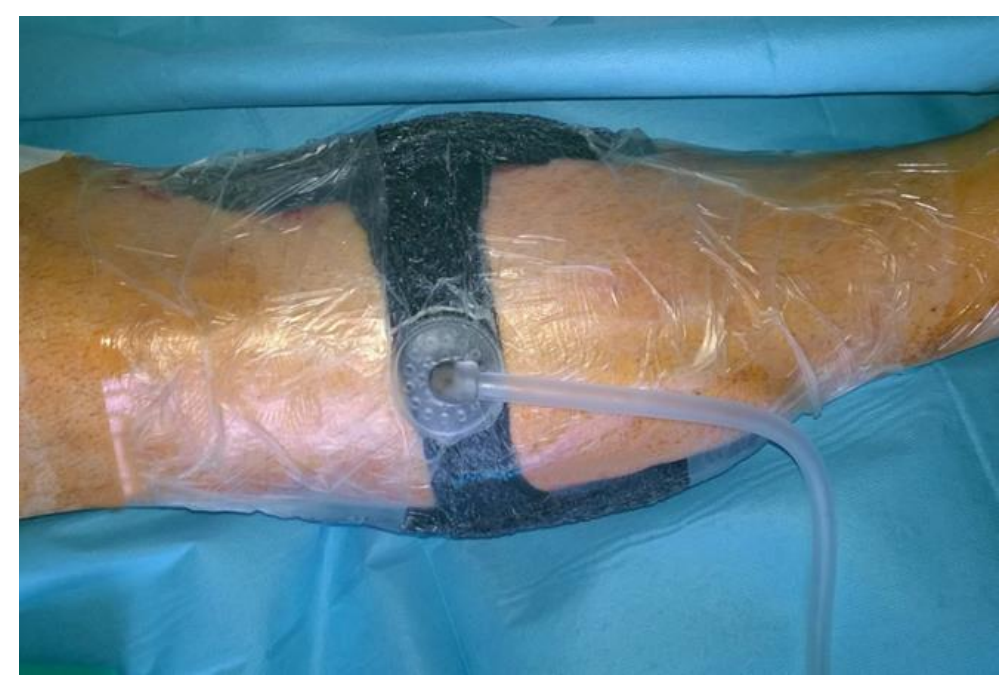

Figure 3. Application of NPWT on the fasciotomy wounds (lateral and medial cut). 
The first approximation of fasciotomy wounds margins occurred on the 4th day after surgery among 67\% of individuals [Fig. 4,5]. The amount of drained fluid from the fasciotomy wound was assessed each time before the replacement of dressing [Fig. 6]. On average, 300-400ml of edema fluid was collected every 3 days. Only in two cases, the amount of fluid was $1000 \mathrm{ml}$.

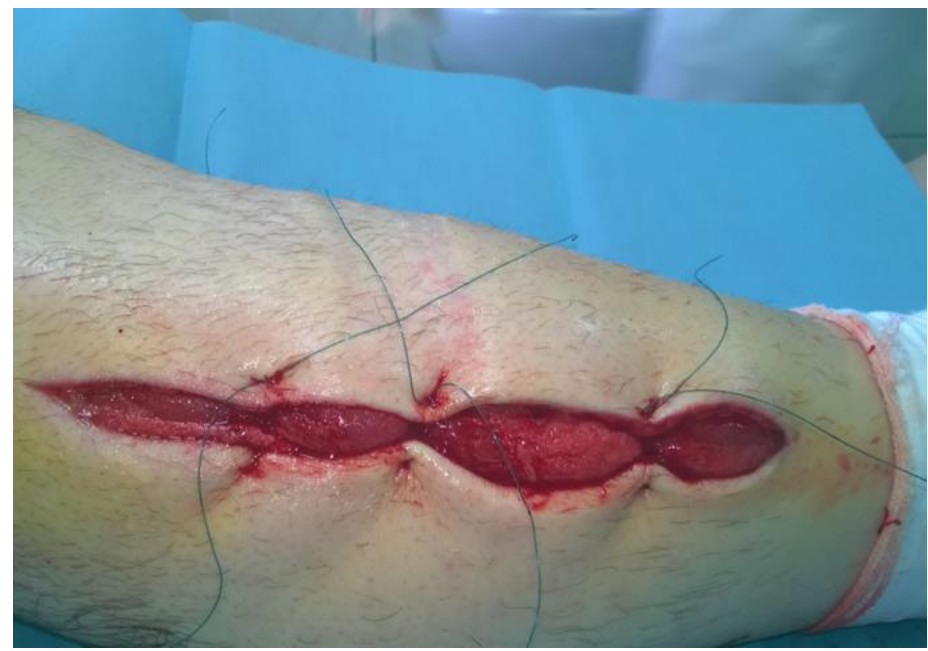

Figure 4. Case 1- the lower limb wound on the 4th day after fasciotomy. Delayed primary closure with sutures - lateral cut.

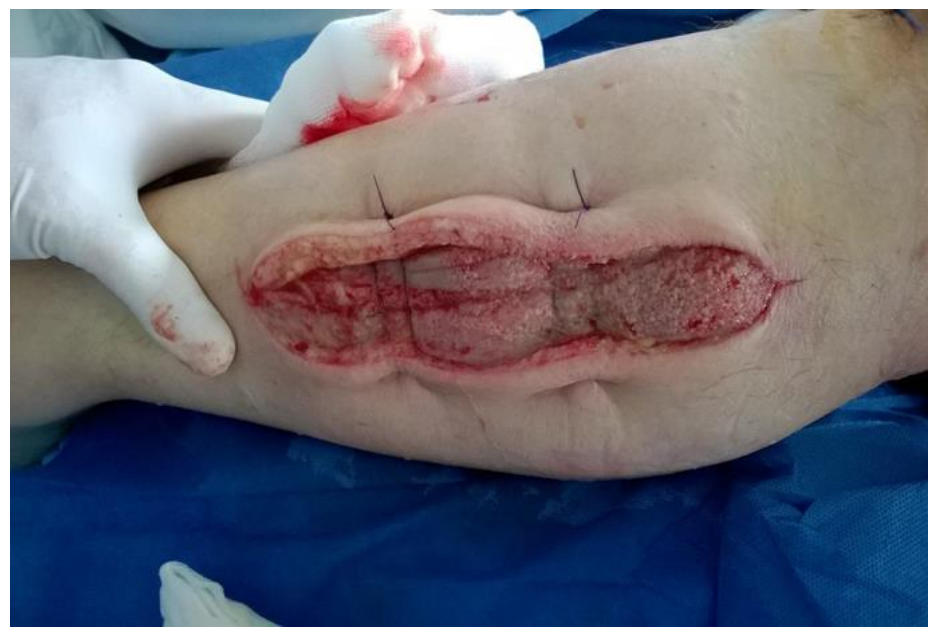

Figure 5. Case 2 - the lower limb wound on the 4 th day after fasciotomy. Delayed primary closure with sutures - lateral cut.

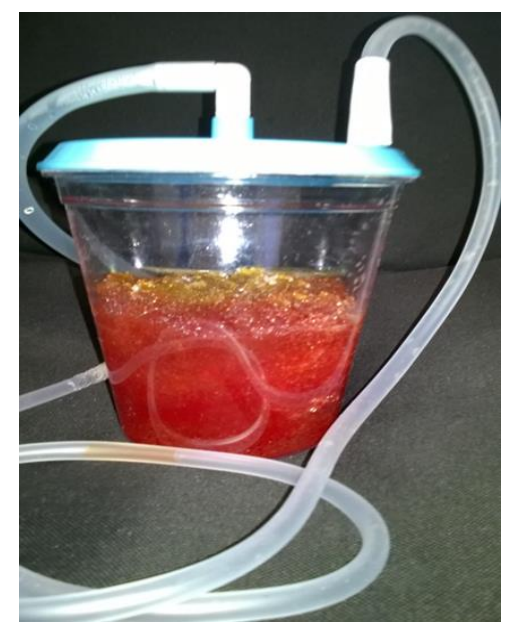

Figure 6. Mean amount of drained fluid within 3 days of applying VAC. 
The main observed clinical effects of the VAC method were the promotion of granulation tissue, reduction of wound edema and reduction excess of fluids. A healing process was completed by surgical closure [Fig. 7-8]. In our group, the average time to definitive closure edges of fasciotomy wounds was 12 days [Fig. 9]. Only in one case the finally closed of wound edges was impossible because of high tissue tension. For this patient skin graft was performed.

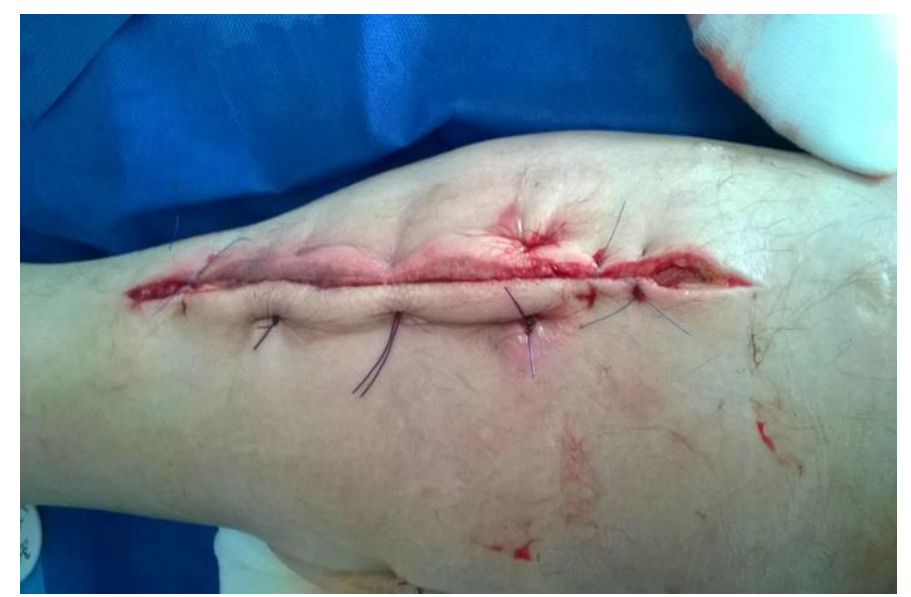

Figure 7. Closure of wound edges on the 8th day after fasciotomy - medial cut.

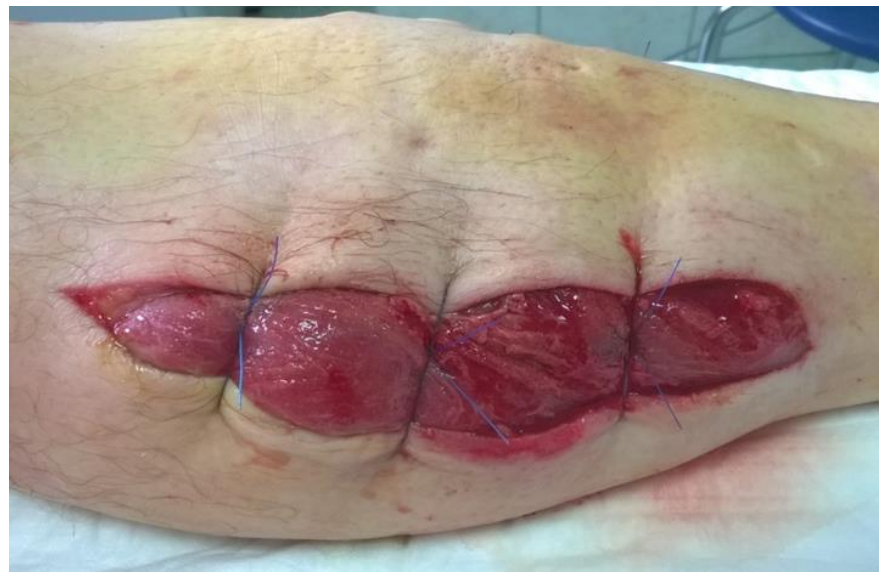

Figure 8. Case 3 - closure of wound edges on the 8th day after fasciotomy - medial cut.

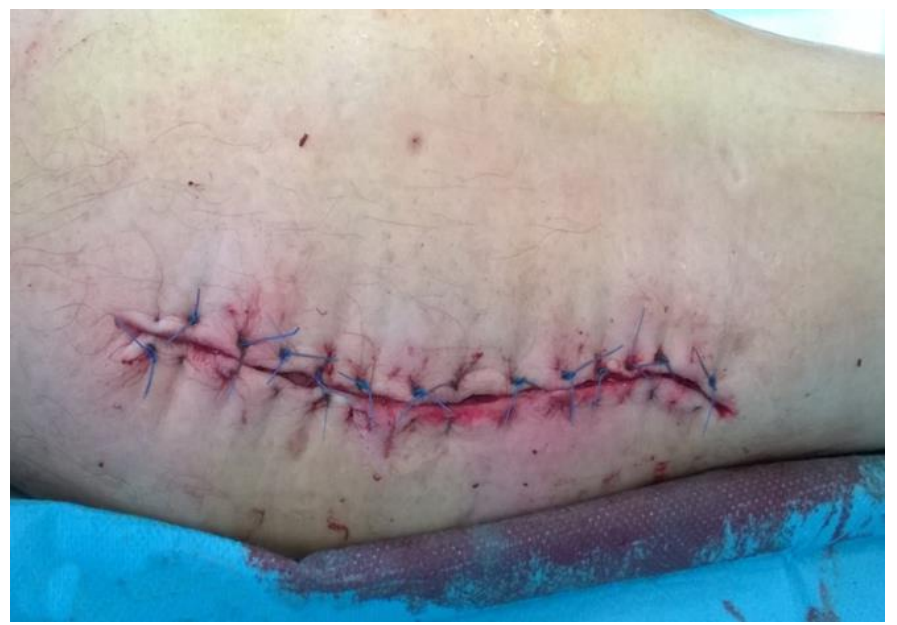

Figure 9. Case 3 - definitive closure of the wound on the 12th day after fasciotomy - medial cut. 
In our study population, the average NPWT use time was 9 days. In the case of correct wounds healing process and good general condition patients were discharged from the hospital with a recommendation to appear for the follow-up examination in the Department of Vascular Surgery. The average hospital stay was 17 days.

After discharge patients from the hospital, a special dressing with ionic silver and foam dressing with a hydrogel layer was recommended for wound management. The follow-up examination revealed further progress of the wound healing. The correct wound healing process allowed to extend time periods between the follow-up visits in the Department of Vascular Surgery [Fig. 10,11].

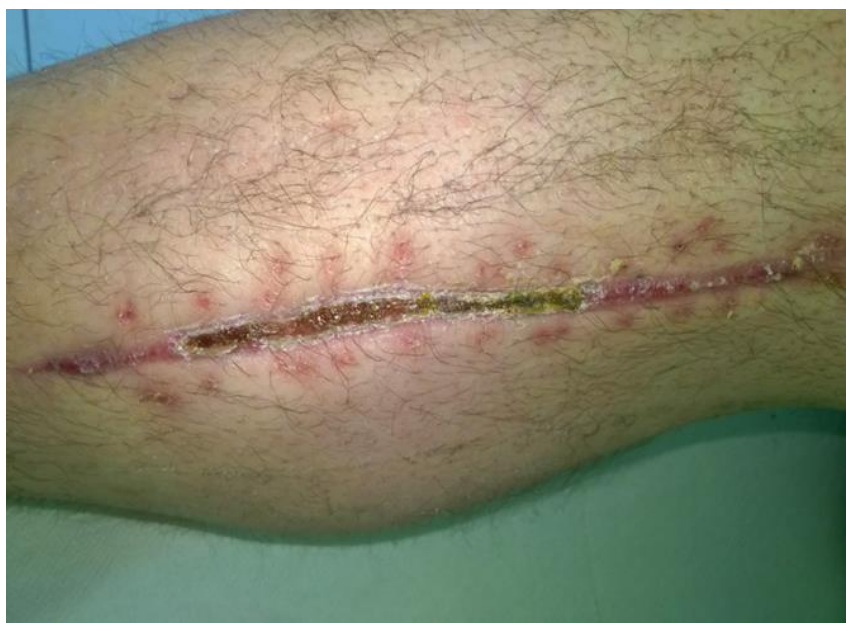

Figure 10. Post-surgery wound on 49th day after fasciotomy - medial cut.

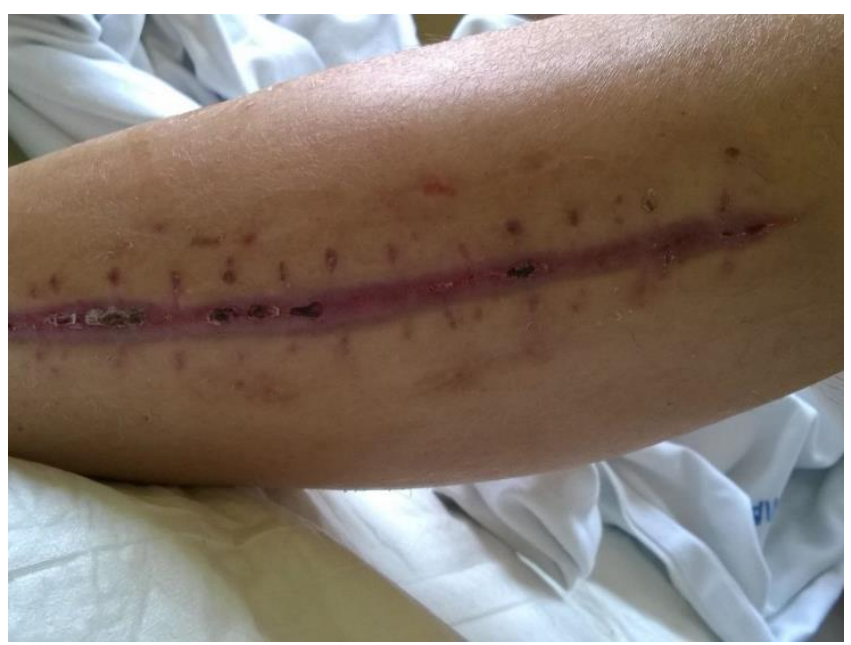

Figure 11. Post-surgery wound on 38th day after fasciotomy - lateral cut.

\section{Discussion}

A crucial role in the management of ACS is early diagnosis and urgent treatment implementation because untreated causes lead to irreversible nerve and muscle ischemia and in consequence, to disabilities. Standardized diagnostic protocols and wound treatment strategies are needed to improved outcomes from this complication. Currently, surgical decompressive fasciotomy is widely recommended [10]. The analysis of single-incision versus dual-incision fasciotomy shows that the results of applied fasciotomy techniques are similar, and the choice of the method should be based on surgeon experience and clinical patients' condition [11]. Unfortunately, primary wound closure might be difficult to achieve due to significant swelling of the limb, skin retraction and skin edge necrosis [12,13]. 
In clinical practice, the NPWT has been obtained an important role in the management of wounds [14]. Effective treatment, low incidence of SSI, positive cosmetic effect and cost-effectiveness have supported the widespread choice of this method therapy. Furthermore, NPWT promotes granulation tissue formation, improves microcirculation and shortens hospitalization period [15-17]. Indications to apply NPWT involve hard to heal wounds including diabetic foot ulcers, traumatic and post-operative wounds, wounds with necrotizing fasciitis [18].

Numerous observational studies support the use of the NPWT in wound management after therapeutic fasciotomy due to ACS. In a retrospective study with a 10-year follow up, Zannis at al. showed that the VAC technique applied to the fasciotomy wounds significantly improved the ability to primary wound closure and relevantly decreased hospitalization time. In this study, the time needed for primary wound closure in the VAC group patients was considerably shorter (8.9 days) than in the group of patients with traditional wet-to-dry dressings (12.7 days) [19]. Similarly, Yang et al. showed that VAC installation decreased time needed to closure edges of wounds after fasciotomy (VAC group 6.7 days vs non-VAC group 16.1 days) [20]. Saziye Karaca et al. in the retrospective study compared the VAC treatment with the conservative treatment of the fasciotomy wound till the time of definitive surgical wound closure. In the study population, fasciotomies were performed because of ischemia-reperfusion syndrome. Noteworthy, the study showed that due to the positive influence on the wound healing process VAC therapy can be a new standard for the treatment of fasciotomy wounds [21].

Finally, our study also has confirmed the beneficial therapeutic effects of NPWT in wound treatment after fasciotomy in ACS. The VAC therapy has led to reduce tissue edema and infection risk, evacuate excess of fluids, as well as promote granulation of tissue. Documented clinical effects have allowed for faster wound closure and shorter hospitalization time, thereby reducing treatment costs.

\section{Conclusions}

ACS is a serious complication that could significantly worsen prognosis in post-reperfusion condition. Our experience and literature review indicate the legitimacy of using NPWT in wound treatment after fasciotomy in ACS. The NPWT used in the treatment of fasciotomy wounds enables faster primary closure of wounds, reduces infection risk and edema as well as decreases hospitalization time

Author Contributions: Conceptualization, J. P. M.; methodology, M. B-S.; validation, J.P.M, M.B-S. A.M. and B.S.; investigation, M.B-S.; writing-original draft preparation, A.M.; writing-review and editing; J.P.M., A.M., B.S. and M.B-S.; visualization, A.M.; supervision, J.P.M.; project administration, J.P.M.

All authors have read and agreed to the published version of the manuscript.

Funding: This research received no external funding. Publication costs will be covered by Collegium Medicum, The Jan Kochanowski University, Kielce, Poland

Conflicts of Interest: The authors declare no conflict of interest.

\section{References}

1. Rothenberg KA, George EL, Trickey AW, Chandra V, Stern JR. Delayed Fasciotomy Is Associated with Higher Risk of Major Amputation in Patients with Acute Limb Ischemia. Ann Vasc Surg. 2019; 195-201. doi: 10.1016/j.avsg.2019.01.028.

2. Rezk F, ?strand H, Acosta S. Incisional negative pressure wound therapy for the prevention of surgical site infection after open lower limb revascularization - Rationale and design of a multi-center randomized controlled trial. Contemp Clin Trials Commun. 2019;16:100469. doi: 10.1016/j.conctc.2019.100469. 
3. Via AG, Oliva F, Spoliti M, Maffulli N. Acute compartment syndrome. Muscles Ligaments Tendons J. 2015;5(1):18-22.

4. Lee P. Treating Fasciotomy Wounds with Negative Pressure Wound Therapy with Instillation and Dwell Time (NPWTi-d). Cureus. 2016 ;8(10):e852.

5. Pechar J, Lyons MM. Acute Compartment Syndrome of the Lower Leg: A Review. J Nurse Pract. 2016;12(4):265-270. doi: 10.1016/j.nurpra.2015.10.013.

6. Bowyer MW. Compartment Syndrome. In: Gahtan V, Costanza MJ, editors. Essentials of Vascular Surgery for the General Surgeon. Springer; 2014. pp. 55-69.

7. Jones DA, Neves Filho WV, Guimar?es JS, Castro DA, Ferracini AM. The use of negative pressure wound therapy in the treatment of infected wounds. Case studies. Rev Bras Ortop. 2016;51(6):646-651. doi:10.1016/j.rboe.2016.10.014

8. Trueman P. Cost-effectiveness considerations for home health V.A.C. therapy in the United States of America and its potential international application. International Wound Journal. 2008;5, supplement 2:23-26.

9. Peinemann F, Labeit A. Negative pressure wound therapy: A systematic review of randomized controlled trials from 2000 to 2017. J Evid Based Med. 2019;12(2):125-132. doi: 10.1111/jebm.12324.

10. Hayakawa H, Aldington DJ, Moore RA. Acute traumatic compartment syndrome: a systematic review of results of fasciotomy. Trauma. 2009; 11(1):5-35

11. Bible JE, McClure DJ, Mir HR. Analysis of single-incision versus dual-incision fasciotomy for tibial fractures with acute compartment syndrome. J Orthop Trauma. 2013 Nov;27(11):607-11. doi: 10.1097/BOT.0b013e318291f284.

12. Krticka M, Ira D, Bilik A, Rotschein P, Svancara J. Fasciotomy closure using negative pressure wound therapy in lower leg compartment syndrome. Bratisl Lek Listy. 2016;117(12):710-714. doi: 10.4149/BLL_2016_136.

13. Fluieraru S, Bekara F, Naud M, Herlin C, Faure C, Trial C, Téot L. Sterile-water negative pressure instillation therapy for complex wounds and NPWT failures. J Wound Care. 2013;22(6):293-4, 296, 2989.

14. Gupta S, Gabriel A, Lantis J, Téot L. Clinical recommendations and practical guide for negative pressure wound therapy with instillation. Int Wound J. 2016;13(2):159-74. doi: 10.1111/iwj.12452.

15. Acosta S, Björck M, Wanhainen A. Negative-pressure wound therapy for prevention and treatment of surgical-site infections after vascular surgery. Br J Surg. 2017;104(2):e75-e84. doi: 10.1002/bjs.10403.

16. Monsen C, Acosta S, Mani K, Wann-Hansson C. A randomised study of NPWT closure versus alginate dressings in peri-vascular groin infections: quality of life, pain and cost. J Wound Care. 2015;24(6):252, 254-6, 258-0. doi: 10.12968/jowc.2015.24.6.252.

17. Lee P. Treating Fasciotomy Wounds with Negative Pressure Wound Therapy with Instillation and Dwell Time (NPWTi-d). Cureus. 2016;8(10):e852.

18. Bernstein, B.H., Tam, H. Combination of subatmospheric pressure dressing and gravity feed antibiotic instillation in the treatment of post-surgical diabetic foot wounds: a case series. Wounds. 2005; 17: 2, 3748

19. Zannis J, Angobaldo J, Marks M, DeFranzo A, David L, Molnar J, Argenta L. Comparison of fasciotomy wound closures using traditional dressing changes and the vacuum-assisted closure device. Ann Plast Surg. 2009; 62(4): 407-9. doi:10.1097/SAP.0b013e3181881b29.

20. Yang CC, Chang DS, Webb LX. Vacuum-assisted closure for fasciotomy wounds following compartment syndrome of the leg. J Surg Orthop Adv. 2006;15(1):19-23.

21. Saziye K, Mustafa C, Ilker U, Afksendyios K. Comparison of vacuum-assisted closure device and conservative treatment for fasciotomy wound healing in ischaemia-reperfusion syndrome: preliminary results. Int Wound J. 2011;8(3):229-36. doi: 10.1111/j.1742-481X.2011.00773.x. 